\title{
A model for control of HIV/AIDS with parental care
}

\author{
Gbenga Jacob Abiodun, Nizar Marcus, Kazeem Oare Okosun and Peter Joseph Witbooi
}

\begin{abstract}
:
In this study we investigate the HIV/AIDS epidemic in a population which experiences a significant flow of immigrants. We derive and analyze a mathematical model that describes the dynamics of HIV infection among the immigrant youths and how parental care can minimize or prevent the spread of the disease in the population. We analyze the model with both screening control and parental care, then investigate its stability and sensitivity behavior. We also conduct both qualitative and quantitative analyses. It is observed that in the absence of infected youths, diseasefree equilibrium is achievable and is globally asymptotically stable. We establish optimal strategies for the control of the disease with screening and parental care, and provide numerical simulations to illustrate the analytic results.
\end{abstract}

\section{Introduction}

It is estimated that HIV spreads at the rate of 7000 people per day worldwide. It has killed more than 30 million people in the last 30 years [19]. Apart from inducing unbearable illness that kills people prematurely, HIV devastates families and communities. The epidemic continues to increase in numbers, most rapidly in Africa and Asia where availability of antiretroviral therapy and health care is seriously inadequate. It also spreads among the youths and teenagers which thus poses an extraordinary risk to life expectancy. There is no established vaccine yet for HIV/AIDS and it is not likely that any highly effective one will soon be available despite all the vigorous studies on vaccination and treatment of the disease.

Mathematical models of transmission dynamics of HIV have been used extensively as a means of informing control strategies, or at least their impact, since they provide short and long term prediction of HIV and AIDS incidence. From the initial models of May and Anderson [2, 3, 11], several modifications have been introduced for studying the extent to which certain factors can play a role in the increase or decrease of HIV prevalence. An investigation on the potential effects and bene- fits of educational campaigns on HIV/AIDS transmission dynamics is undertaken in $[5,16,18]$ and other papers. Other papers study the effect of condom use [7], and other factors impacting on HIV. However, the authors are not aware of mathematical models in the literature that include the effect of parental care and its optimal control. This paper is an attempt towards filling this gap. A very handy reference on application of optimal control theory to epidemiology is the book of Lenhart and Workman [10]. There are 
numerous studies on epidemiological models where optimal control methods were applied.

It has been pointed out that parental care has a substantial effect on the spread of AIDS among the youths. Parental care means bringing up a child in a decent manner, providing for their moral, material, financial needs and giving them quality education. Parental care also involves providing children with sex education and introducing them to the available health educational campaigns where they can learn about transmission of different diseases and how they can be protected. Research clearly shows that a child, who lacks adequate parental instruction, stands at risk of recalcitrance. A certain study [8] on 94,000 cases of child delinquent behavior reveals that about $80 \%$ of such cases stem from children in households with poor parental care and counseling.

Our objective is to show how parental care could reduce the spread of HIV/AIDS among the youth. In Sec. 2 of this paper we present the HIV model. The proof of global stability of the disease-free equilibrium follows in Sec. 3. In Sec. 4 we present a discussion of the endemic equilibrium point. The sensitivity analysis of the basic reproduction ratio is presented in Sec. 5. In Sec. 6 we present the optimal control problem together with its solution. Numerical results and analysis of the control problem appears in Sec. 7, while Sec. 8 offers some concluding remarks.

\section{HIV/AIDS Model Formulation}

We propose a model which is depicted in the flow diagram below. The total population at time $t$, denoted by $N(t)$ is sub-divided into sub-populations of susceptible individuals $(S(t))$, individuals newly infected with HIV $(I(t))$, individuals with HIV but not yet developed to $\operatorname{AIDS}(H(t))$, and individuals with $\operatorname{AIDS}(A(t))$. The population size is $N(t)$ and

$$
N(t)=S(t)+I(t)+H(t)+A(t) .
$$

The natural and AIDS induced death rates are $\mu$ and $\alpha$ respectively. Susceptible individuals are recruited at a rate $\left(1-\rho u_{1}\right) \mu K$ where $\rho$ is the proportion of infectious youths and $u_{1} \in[0,1]$ is the effort of screening control on immigrant youths (a quarantine se). The susceptible acquire HIV by any blood contact with infectious youths at a rate

$$
\left(1-u_{2}\right) \lambda S, \quad \text { with } \quad \lambda=\beta_{1} c_{1} I+\beta_{2} c_{2} H+\beta_{3} c_{3} A,
$$

where $u_{2}$ accounts for parental care and $\beta_{1}, \beta_{2}, \beta_{3}$ are transmission probabilities. The progression rate from newly infected individuals to HIV is $\gamma$ and that of HIV to AIDS is $\sigma$. The number of partners with newly infected individuals, individuals having HIV, and individuals having AIDS are $c_{1}, c_{2}$ and $c_{3}$ respectively. 
We further assume that parental care involves all efforts and activities taken by parents to prevent their children from contracting HIV. The resulting system of

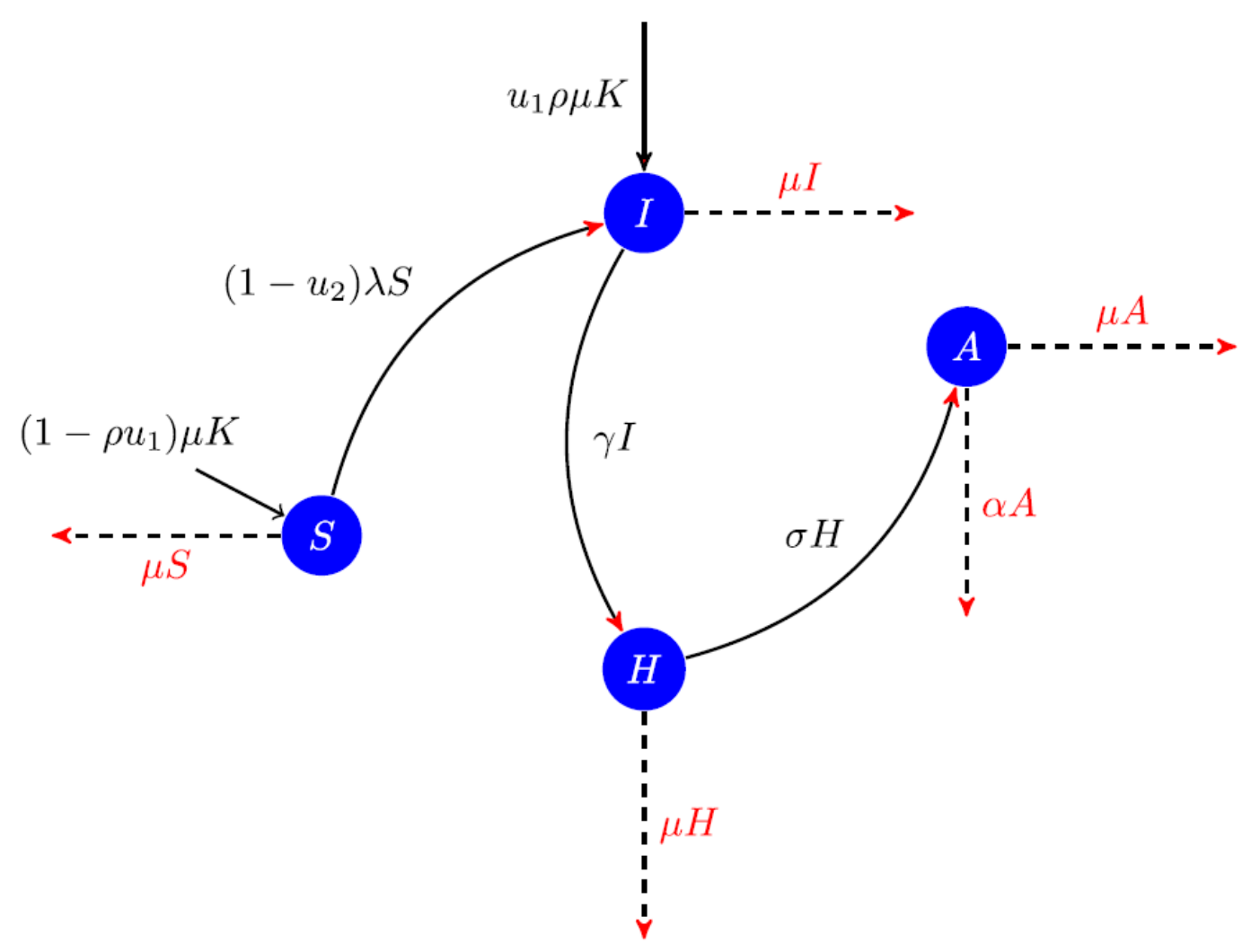

Fig. 1. Flow diagram for HIV/AIDS transmission.

equations is shown below:

$$
\left\{\begin{array}{l}
\frac{d S}{d t}=\left(1-\rho u_{1}\right) \mu K-S\left(1-u_{2}\right)\left(I c_{1} \beta_{1}+H c_{2} \beta_{2}+A c_{3} \beta_{3}\right)-\mu S \\
\frac{d I}{d t}=\rho u_{1} \mu K+S\left(1-u_{2}\right)\left(I c_{1} \beta_{1}+H c_{2} \beta_{2}+A c_{3} \beta_{3}\right)-(\gamma+\mu) I \\
\frac{d H}{d t}=\gamma I-(\mu+\sigma) H \\
\frac{d A}{d t}=\sigma H-(\alpha+\mu) A .
\end{array}\right.
$$

To make a model to be closer to the real life, we assume that all other classes of individuals except susceptible are infectious and that the number of infectious individuals, that is, the sum $I(t)+H(t)+A(t)$, is less than the number of susceptible, $S(t)$. 


\section{Stability of the Disease-Free Equilibrium}

\section{- Local stability of the disease-free equilibrium}

In the absence of infected and infectious youths entering the population, that is when $\rho$ $=0$, we establish the stability of the diseases free equilibrium (DFE), $E_{0}$. The DFE of the HIV model (2.1) is the point $E_{\mathrm{O}}=(K, 0,0,0)$.

Theorem 3.1. The basic reproductive number $R_{0}$ of model (2.1) is given by

$$
R_{0}=\frac{K\left(1-u_{2}\right)\left((\alpha+\mu)\left((\mu+\sigma) c_{1} \beta_{1}+\gamma c_{2} \beta_{2}\right)+\gamma \sigma c_{3} \beta_{3}\right)}{(\alpha+\mu)(\gamma+\mu)(\mu+\sigma)}
$$

Proof. Following van den Driessche and Watmough [20], the basic reproductive number $R$ of model (2.1) is calculated by using the next generation matrix. It is given by

$$
R_{0}=r\left(F V^{-1}\right),
$$

where $r(\cdot)$ denotes the spectral radius, with

$$
F=\left(\begin{array}{ccc}
\beta_{1} c_{1}\left(1-u_{2}\right) K & \beta_{2} c_{2}\left(1-u_{2}\right) K & \beta_{3} c_{3}\left(1-u_{2}\right) K \\
0 & 0 & 0 \\
0 & 0 & 0
\end{array}\right)
$$

And

$$
V^{-1}=\left(\begin{array}{ccc}
\frac{1}{\gamma+\mu} & 0 & 0 \\
\frac{\alpha \gamma+\gamma \mu}{(\alpha+\mu)(\gamma+\mu)(\mu+\sigma)} & \frac{1}{\mu+\sigma} & 0 \\
\frac{\gamma \sigma}{(\alpha+\mu)(\gamma+\mu)(\mu+\sigma)} & \frac{\gamma \sigma+\mu \sigma}{(\alpha+\mu)(\gamma+\mu)(\mu+\sigma)} & \frac{1}{\alpha+\mu}
\end{array}\right) .
$$

Thenumerical value of $R_{O}$ can be routinely calculated as asserted.

Theorem 3.2. The disease-free equilibrium $E_{\mathrm{O}}$, exists for all $R_{\mathrm{O}}$, and is locally asymptotically stable for $R_{\mathrm{O}}<1$ and unstable if $R>1$. 


\subsection{Global stability of disease-free equilibrium $E_{O}$}

We prove global stability of the DFE subject to a certain condition that $\mu+\sigma<1$, which is quite reasonable anyway.

Theorem 3.3. Suppose that in model (2.1), we take $\rho=0$ and assume that $\mu+\sigma<1$. Then the equilibrium point $E_{\mathrm{O}}$ is globally asymptotically stable when $R_{\mathrm{O}}<1$.

Proof. In order to construct a Lyapunov function, we introduce the following positive constants $x$ and $y$. Let

$$
x=\frac{\alpha+\mu}{c_{3} \beta_{3} K\left(1-u_{2}\right)} \quad \text { and } \quad y=\frac{c_{3} \beta_{3} \sigma+c_{2} \beta_{2}(\mu+\alpha)}{c_{3} \beta_{3}(\mu+\sigma)}
$$

Now we define $V(t)$ to be the function

$$
V(t)=x I(t)+y H(t)+A(t) .
$$

In what follows we prove that $\dot{V}(t)$ is a negative-definite function in the variable $(I(t), H(t)$, $A(t))$. First we note that $\dot{V}(t)$ can be expressed in the form

$$
\dot{V}(t)=Q_{I} I(t)+Q_{H} H(t)+Q_{A} A(t),
$$

where the coefficients $Q I, Q H$ and $Q A$ are as follows:

$$
\begin{aligned}
Q_{I} & =x S\left(1-u_{2}\right) c_{1} \beta_{1}-x(\mu+\gamma)+y \gamma \\
Q_{H} & =x S\left(1-u_{2}\right) c_{2} \beta_{2}-y(\mu+\sigma)+\sigma, \\
Q_{A} & =x S\left(1-u_{2}\right) c_{3} \beta_{3}-(\mu+\alpha) .
\end{aligned}
$$

We also note that $S<K$. Now we prove that the coefficients $Q I, Q H$ and $Q A$ are negative. First we note that with $M=K\left(1-u_{2}\right)$, we obtain

$$
Q_{A}<x M c_{3} \beta_{3}-(\mu+\alpha)=0 .
$$


Next we note that

$$
\begin{aligned}
Q_{H} & <x M c_{2} \beta_{2}-y(\mu+\sigma)+\sigma \\
& =\frac{c_{2} \beta_{2}(\mu+\alpha)(\mu+\sigma)-c_{2} \beta_{2}(\mu+\alpha)-c_{3} \beta_{3} \sigma+c_{3} \beta_{3} \sigma(\mu+\sigma)}{c_{3} \beta_{3}(\mu+\sigma)} \\
& =\frac{c_{2} \beta_{2}(\mu+\alpha)[\mu+\sigma-1]+c_{3} \beta_{3} \sigma(\mu+\sigma-1)}{c_{3} \beta_{3}(\mu+\sigma)} \\
& =\frac{(\mu+\sigma-1)\left(c_{2} \beta_{2}(\mu+\alpha)+c_{3} \beta_{3}\right)}{c_{3} \beta_{3}(\mu+\sigma)}<0, \quad \text { since } \mu+\sigma-1<0 .
\end{aligned}
$$

Finally

$$
\begin{aligned}
Q_{I} \leq & x M c_{1} \beta_{1}+y \gamma-x(\mu+\gamma) \\
= & \frac{M c_{1} \beta_{1}(\mu+\alpha)}{M c_{3} \beta_{3}}+\gamma \frac{c_{3} \beta_{3} \sigma+c_{2} \beta_{2}(\mu+\alpha)}{c_{3} \beta_{3}(\mu+\sigma)}-\frac{(\mu+\alpha)(\mu+\sigma)}{c_{3} \beta_{3} M} \\
= & \frac{1}{M c_{3} \beta_{3}(\mu+\sigma)}\left[M c_{1} \beta_{1}(\mu+\alpha)(\mu+\sigma)+M \gamma\left(c_{3} \beta_{3} \sigma+c_{2} \beta_{2}(\mu+\alpha)\right)\right. \\
& -(\mu+\alpha)(\mu+\gamma)(\mu+\sigma)] \\
= & \frac{(\mu+\alpha)(\mu+\gamma)}{M c_{3} \beta_{3}} \\
& \times\left[\frac{M c_{1} \beta_{1}(\mu+\alpha)(\mu+\sigma)+M \gamma\left(c_{3} \beta_{3} \sigma+c_{2} \beta_{2}(\mu+\alpha)\right)+M c_{3} \beta_{3} \gamma \sigma}{(\mu+\alpha)(\mu+\gamma)(\mu+\sigma)}-1\right] \\
= & \frac{(\mu+\alpha)(\mu+\gamma)}{M c_{3} \beta_{3}}\left[R_{0}-1\right]<0 .
\end{aligned}
$$

This completes the proof.

\section{Existence of Endemic Equilibrium}

In search of an endemic equilibrium point, we arrive at the following point $E_{1}$ : 


$$
\left\{\begin{aligned}
S^{*} & =\frac{K \mu\left(1-\rho u_{1}\right)}{\lambda+\mu-\lambda u_{2}}, \\
I^{*} & =\frac{K \lambda \mu+K \mu^{2} \rho u_{1}-K \lambda \mu u_{2}}{(\gamma+\mu)\left(\lambda+\mu-\lambda u_{2}\right)} \\
H^{*} & =\frac{\gamma\left(-(K \lambda \mu)-K \mu^{2} \rho u_{1}+K \lambda \mu u_{2}\right)}{(\gamma+\mu)(\mu+\sigma)\left(\lambda+\mu-\lambda u_{2}\right)} \\
A^{*} & =\frac{\gamma \sigma\left(-(K \lambda \mu)-K \mu^{2} \rho u_{1}+K \lambda \mu u_{2}\right)}{(\alpha+\mu)(\gamma+\mu)(\mu+\sigma)\left(\lambda+\mu-\lambda u_{2}\right)} .
\end{aligned}\right.
$$

The number $\lambda$ is as defined earlier, in the system of Eqs. (2.1). At the same time, $\lambda$ is a root of the following polynomial (and as in the proof of Theorem 3.3 we set $M=K(1-$ $\left.u_{2}\right)$ ):

$$
W_{1} \lambda^{2}+W_{2} \lambda+W_{3}=0
$$

With

$$
\begin{aligned}
W_{1}= & (\alpha+\mu)(\gamma+\mu)(\mu+\sigma)\left(1-u_{2}\right), \\
W_{2}= & \mu((\alpha+\mu)(\gamma+\mu)(\mu+\sigma) \\
& \left.-M\left((\alpha+\mu)\left((\mu+\sigma) c_{1} \beta_{1}+\gamma c_{2} \beta_{2}\right)+\gamma \sigma c_{3} \beta_{3}\right)\right), \\
W_{3}= & -\left(K \mu^{2} \rho u_{1}\left((\alpha+\mu)\left((\mu+\sigma) c_{1} \beta_{1}+\gamma c_{2} \beta_{2}\right)+\gamma \sigma c_{3} \beta_{3}\right)\right) .
\end{aligned}
$$

Let us write $-W_{2}=\omega$. For the case $\rho=0$, the quadratic equation (4.2) has a root $\lambda_{0}=0$ which corresponds to the disease-free equilibrium and another root

$$
\lambda_{1}=\frac{\mu\left((\alpha+\mu)(\gamma+\mu)(\mu+\sigma)-M\left((\alpha+\mu)\left((\mu+\sigma) c_{1} \beta_{1}+\gamma c_{2} \beta_{2}\right)+\gamma \sigma c_{3} \beta_{3}\right)\right)}{(\alpha+\mu)(\gamma+\mu)(\mu+\sigma)\left(1-u_{2}\right)}
$$

which is positive if and only if $-W_{2}>0$, i.e. if $\omega>0$. At the same time we note that a negative value of $\lambda$ will result in a point $E_{1}$ which is non-feasible. If $\rho>0$ the quadratic equation has one positive root and one negative root. By definition of $\lambda$ we have $\lambda \geq 0$, and therefore we discard the negative root. The positive root is given as 


$$
\lambda^{*}=\frac{-W_{2}+\sqrt{W_{2}^{2}}-4 W_{1} W_{3}}{2 W_{1}} .
$$

We note that $\omega>0$ if and only if $R>1$, and $\omega<0$ if and only if $R_{0}<1$. We observe that

$$
\lim _{\rho \rightarrow 0} \lambda^{*}=\frac{\omega+|\omega|}{2 W_{1}}= \begin{cases}0 & (\omega<0) \\ \frac{\omega}{W_{1}} & (\omega>0) .\end{cases}
$$

This shows that for $\rho$ close to zero, the model has a threshold $R_{\mathrm{O}}=1$. For $\rho>0$, the disease remains endemic, so system (2.1) has one endemic equilibrium point for all parameter values for which the disease will always persist in the population. If $R_{O}<1$ as $\rho \rightarrow \mathrm{o}$, then the endemic equilibrium tends to the disease-free equilibrium. Otherwise if $R_{\mathrm{O}}>1$ as $\rho \rightarrow \mathrm{O}$, then the model has a unique endemic equilibrium.

\section{Sensitivity Analysis of $R_{0}$}

We carry out sensitivity analysis to investigate the robustness of the basic reproductive number $R_{O}$ to the parameters. This will help us to identify the parameters that have high impact on the diseases transmission, that is, on the reproductive number. One way to derive a sensitivity index is by using partial derivatives when the variable is a differentiable function of the parameter.

Definition. The normalized forward sensitivity index of a variable, $m$, that depends differentiably on a parameter, $n$, is defined as:

$$
\Upsilon_{n}^{m}=\frac{\partial m}{\partial n} \times \frac{n}{m} .
$$

Wederive the sensitivity of $R_{\mathrm{O}}$ to each of the parameters described in Table 1.

Thesensitivityindex is shown below for the parameter $\sigma$.

$$
\frac{\partial R_{0}}{\partial \sigma} \times \frac{\sigma}{R_{0}}=\frac{\gamma \sigma\left((-\alpha-\mu) c_{2} \beta_{2}+\mu c_{3} \beta_{3}\right)}{(\mu+\sigma)\left((\alpha+\mu)\left((\mu+\sigma) c_{1} \beta_{1}+\gamma c_{2} \beta_{2}\right)+\gamma \sigma c_{3} \beta_{3}\right)} .
$$

The indices with respect to the other model parameters can be calculated similarly. We omit the detail. 
Table 1. Parameter values and sensitivity indices of $R_{0}$.

\begin{tabular}{clccl}
\hline Parameter & \multicolumn{1}{c}{ Parameter description } & Estimated value & References & Sensitivity \\
\hline$c_{3}$ & Number of partners with AIDS & 1 & Nominal & +0.795 \\
$\beta_{3}$ & Trans. prob. of AIDS & $0 \leq \beta_{3} \leq 1$ & {$[18]$} & +0.795 \\
$\mu$ & Natural death rate of individual & 0.02 & {$[6,12-14]$} & -0.6696 \\
$\alpha$ & AIDS induced death rate & 0.33 & {$[13-15,18]$} & +0.159 \\
$c_{1}$ & Num. of partners with $I$ indiv. & 4 & Nominal & +0.159 \\
$\beta_{1}$ & Trans. prob. of Infected & $0 \leq \beta_{1} \leq 1$ & {$[18]$} & +0.159 \\
$\gamma$ & Progression rate from $S$ to $I$ & 0.18 & {$[18]$} & -0.1428 \\
$c_{2}$ & Num. of partners with HIV & 3 & Nominal & +0.045 \\
$\beta_{2}$ & Trans. prob. of HIV & $0 \leq \beta_{2} \leq 1$ & {$[18]$} & +0.045 \\
$\sigma$ & Progression rate from $H$ to $A$ & 0.05 & {$[18]$} & -0.0286 \\
\hline
\end{tabular}

Parameter values. In Table 1 we list parameter values together with references.

For the parameters $c_{1}, c_{2}$ and $c_{3}$ we supply just nominal values, in line with other literature such as $[4,12,13]$ for instance. Parameters are also arranged from the most sensitive to the least. The most sensitive parameters are the number of partners with AIDS individuals $\left(c_{3}\right)$ and transmission probability of getting AIDS $\left(\beta_{3}\right)$. The least is the progression rate from HIV to AIDS ( $\sigma$ ).

The sensitivity index of $R_{\mathrm{O}}$ with respect to the transmission probability of get- ting AIDS $\left(\beta_{3}\right)$ is +0.795 , implying that decreasing (or increasing) the $\beta_{3}$ by $1 \%$, increases $R_{\mathrm{O}}$ by approximately $0.8 \%$. Similarly increasing (or decreasing) the natural death rate ( $\mu$ ) by $1 \%$, decreases the $R_{0}$ by approximately $0.7 \%$.

For all the parameters, the sign of the sensitivity indices of $R_{\mathrm{O}}$ agrees with the intuitive expectation as to whether $R_{\mathrm{O}}$ increases or decreases when the parameters increases.

\section{Optimal Control Analysis}

In this section we regard the entities $u_{1}$ and $u_{2}$ as functions $u_{1}(t)$ and $u_{2}(t)$ of time, since they are the very controls in question. Recall that $u_{1}$ and $u_{2}$ are related to screening and parental care respectively. Then we apply optimal control method to determine the necessary conditions for the control of HIV/AIDS. In order to investigate the optimal level of parental effort that would be needed to control the disease, we propose an objective function $J$ minimized:

$$
J=\int_{0}^{\tau}\left[Q_{0} H+Q_{1} I+Q_{2} A+Q_{3} u_{1}^{2}+Q_{4} u_{2}^{2}\right] d t .
$$

Here $Q_{0}, Q_{1}, Q_{2}, Q_{3}, Q_{4}$ are positive weights, and we choose quadratic cost on the controls, which is similar to other literature on epidemic controls $[1,9,10]$. With the given objective function $J\left(u_{1}, u_{2}\right)$, our goal is to minimize the numbers in the infected classes, each 
class having its own weight, while also minimizing the cost of control $u_{1}(t), u_{2}(t)$. We choose an optimal control $u * 1(t), u * 2(t)$ such that

$$
J\left(u_{1}^{*}, u_{2}^{*}\right)=\min \left\{J\left(u_{1}, u_{2}\right) \mid u_{1}, u_{2} \in U\right\}
$$

where $U=\left\{\left(u_{1}, u_{2}\right)\right.$ such that $\left(u_{1}(t), u_{2}(t)\right)$ are measurable with $u_{1}, u_{2} \in[0,1]$ for $t \in$ $[0, \tau]\}$ is the control set. The necessary conditions that an optimal control must satisfy come from the Pontryagin's Maximum Principle, see for instance the textbook [10]. This principle converts (2.1) and (6.2) into a problem of minimizing pointwise a Hamiltonian $H$, with respect to $u_{1}$ and $u_{2}$. The Hamiltonian is as follows:

$$
\begin{aligned}
\mathcal{H}= & Q_{0} H+Q_{1} I+Q_{2} A+Q_{3} u_{1}^{2}+Q_{4} u_{2}^{2} \\
& +\eta_{S}\left[\left(1-\rho u_{1}\right) \mu K-S\left(1-u_{2}\right)\left(I c_{1} \beta_{1}+H c_{2} \beta_{2}+A c_{3} \beta_{3}\right)-\mu S\right] \\
& +\eta_{I}\left[\rho u_{1} \mu K+S\left(1-u_{2}\right)\left(I c_{1} \beta_{+} H c_{2} \beta_{2}+A c_{3} \beta_{3}\right)-(\gamma+\mu) I\right] \\
& +\eta_{H}[\gamma I-(\mu+\sigma) H]+\eta_{A}[\sigma H-(\alpha+\mu) A],
\end{aligned}
$$

where $\eta_{S}, \eta_{I}, \eta_{H}$ and $\eta_{A}$ are the adjoint variables. Weapply Pontryagin's Maximum Principle and the existence result for the optimal control from $[10]$. Then for the optimal control $\left(u^{*}\right.$, $\left.u^{*}\right)$ that minimizes $J(u, u)$, the adjoint variables $\eta_{S}, \eta_{I}, \eta_{H}$ and $\eta_{A}$

$$
\begin{aligned}
& -\frac{d \eta_{S}}{d t}=\left(u_{2}-1\right)\left(I c_{1} \beta_{1}+H c_{2} \beta_{2}+A c_{3} \beta_{3}\right)\left(\eta_{I}-\eta_{S}\right)+\mu \eta_{S}, \\
& -\frac{d \eta_{I}}{d t}=-Q_{1}+S c_{1}\left(u_{2}-1\right) \beta_{1}\left(\eta_{I}-\eta_{S}\right)-\gamma \eta_{H}+(\gamma+\mu) \eta_{I}, \\
& -\frac{d \eta_{H}}{d t}=-\left(\sigma \eta_{A}\right)+(\mu+\sigma) \eta_{H}+S c_{2}\left(u_{2}-1\right) \beta_{2}\left(\eta_{I}-\eta_{S}\right), \\
& -\frac{d \eta_{A}}{d t}=-Q_{2}+(\alpha+\mu) \eta_{A}+S c_{3}\left(u_{2}-1\right) \beta_{3}\left(\eta_{I}-\eta_{S}\right)
\end{aligned}
$$

and with transversality conditions

$$
\eta_{S}(\tau)=\eta_{I}(\tau)=\eta_{H}(\tau)=\eta_{A}(\tau)=0
$$


Theorem 6.1. The optimal controls take the form:

$$
\begin{aligned}
& u_{1}^{*}=\max \left\{0, \min \left(1, \frac{\mu \rho K\left(\eta_{S}-\eta_{I}\right)}{2 Q_{3}}\right)\right\}, \\
& u_{2}^{*}=\max \left\{0, \min \left(1, \frac{S\left(I c_{1} \beta_{1}+H c_{2} \beta_{2}+A c_{3} \beta_{3}\right)\left(\eta_{I}-\eta_{S}\right)}{2 Q_{4}}\right)\right\} .
\end{aligned}
$$

Proof. The existence of an optimal control is due to the convexity of the integral of $J$ with respect to $u_{1}, u_{2}, a$ priori boundedness of the state solutions, and the Lipschitz property of the state system with respect to the variables (see for instance [10]). The differential equations governing the adjoint variables are obtained by differentiation of the Hamiltonian function, evaluated at the optimal control. By standard control arguments involving the bounds on the controls, we conclude

$$
\begin{aligned}
& u_{1}^{*}= \begin{cases}0 & \text { if } \xi_{1}^{*} \leq 0, \\
\zeta_{1}^{*} & \text { if } 0<\zeta_{1}^{*}<1, \\
1 & \text { if } \zeta_{1}^{*} \geq 1,\end{cases} \\
& u_{2}^{*}=\left\{\begin{array}{cl}
0 & \text { if } \xi_{1}^{*} \leq 0, \\
\zeta_{2}^{*} & \text { if } 0<\zeta_{2}^{*}<1, \\
1 & \text { if } \zeta_{2}^{*} \geq 1,
\end{array}\right.
\end{aligned}
$$

Where

$$
\begin{aligned}
\zeta_{1}^{*} & =\frac{\mu \rho K\left(\eta_{S}-\eta_{I}\right)}{2 Q_{3}}, \\
\zeta_{2}^{*} & =\frac{S\left(I c_{1} \beta_{1}+H c_{2} \beta_{2}+A c_{3} \beta_{3}\right) \eta_{I}-S\left(I c_{1} \beta_{1}+H c_{2} \beta_{2}+A c_{3} \beta_{3}\right) \eta_{S}}{2 Q_{4}} .
\end{aligned}
$$


This completes the proof.

Due to the boundedness of the state system, adjoint system and the resulting Lipschitz structure of the ODEs, we obtain the uniqueness of the optimal control for small $\tau$. Uniqueness of the optimal control follows from the uniqueness of the optimality system, which consists of (6.1) and (6.2) with characterization (6.3).

\section{Numerical Results and Discussion}

In this section we present some numerical solution to the control problem. An iterative scheme is used for solving the optimality system. Using a fourth-order Runge-Kutta scheme, we start off with a guess for the control over the simulated time. Because of the transversality conditions (6.5), the adjoint equations are solved by a backward fourthorder Runge-Kutta scheme using the current iterations as solutions of the state equation. Then the controls are updated by using a convex combination of the previous controls and the value from the characterizations (6.6). This process is repeated and iteration stopped if the values of the unknowns at the previous iterations are very close to the ones at the present iterations, see [10].

We examine our deterministic model and study the effects of screening control $\left(u_{1}\right)$ and parental care $\left(u_{2}\right)$ on each of the classes. We also investigate numerically the effect of the different optimal control strategies (labeled $A, B$ and $C$ below) on the spread of HIV/AIDS among the youths.

We assume that the weight factor $Q_{4}$, associated with control $u_{2}$ is greater than $Q_{3}$ which is associated with $u_{1}$. This assumption is based on the fact that the cost associated with $u_{1}$ will include the cost of screening and surveillance, while those associated with $u_{2}$ will include education, hospitalization, medical testing and so on. We also assumed that $I$-compartment individuals have more partners than those in $H$ and $A$ since they are much elss active. We choose the set of weight factors $Q_{0}=1, Q_{1}=$ $0.5, Q_{2}=0.3, Q_{3}=0.4$ and initial values $S(0)=85,000, I(0)=10,000, H(0)=$ $5000, A(0)=0$. The parameter values used for simulations are as in Table 1 with $K$ $=100,000, \beta_{1}=0.00000034, \beta_{2}=0.00000023$ and $\beta_{3}=0.00000015$. Thus, we illustrate the effect of different optimal control strategies on the spread of HIV/AIDS among the youths.

\section{Strategy A: Optimal use of screening control $\left(u_{1}\right)$ and parental care $\left(u_{2}\right)$ on individuals.}

The screening control $\left(u_{1}\right)$ and parental care $\left(u_{2}\right)$ are used to optimize the objective function $J$. We observe in Fig. 2(a) that due to control strategies, the number of infected individuals $I$ decreases to zero at time $t=30$ while the population of infectious reduces to zero at $t=70$ when there is no control. In Fig. 2(b), the number of HIV individuals first increases from initial 5000 to almost 8000 but later 
decreases to zero at $t=70$ when there is control. It rapidly increases to more than 11,000 before reducing to 200 at $t=80$ when there is no control. AIDS individuals in Fig. 2(c) increases from initially o to 1000 and decreases back to zero at $t=80$,

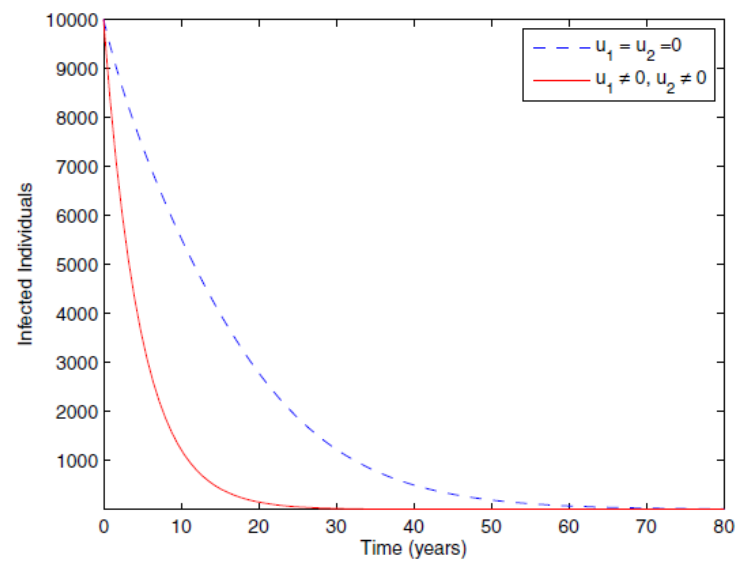

(a)

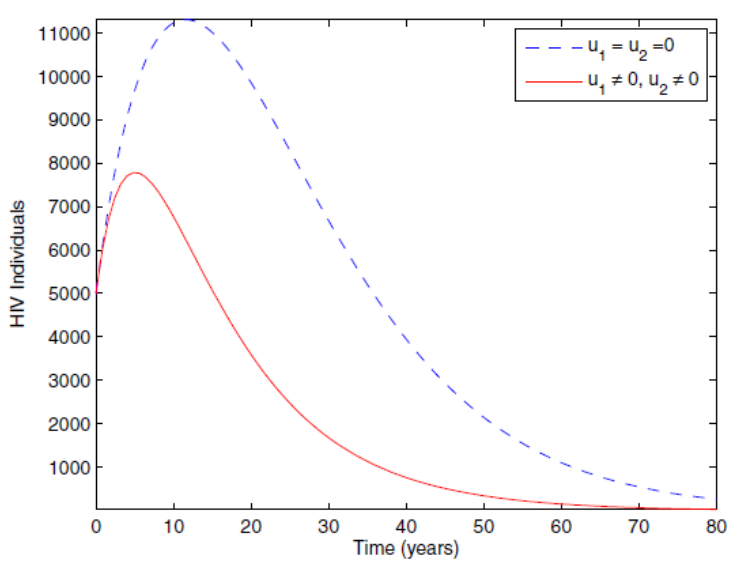

(b)

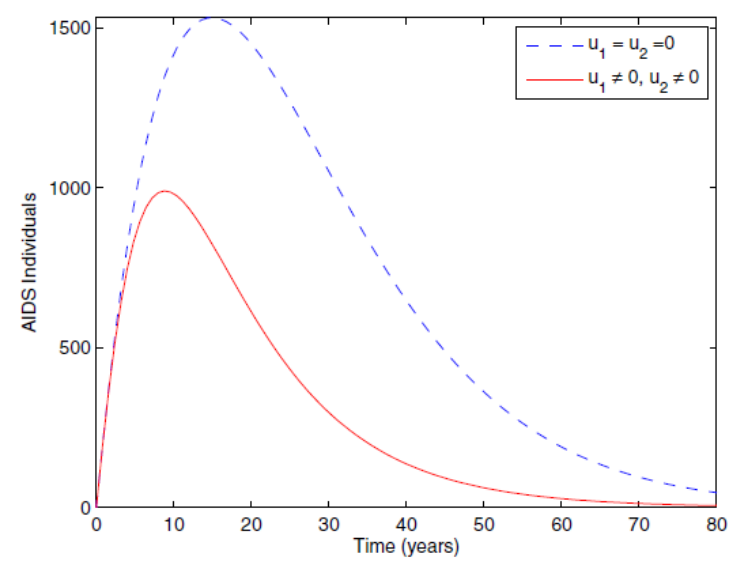

(c)

Fig. 2. Simulations of HIV/AIDS model showing the effect of optimal control strategies using screening control and parental care on individuals. 


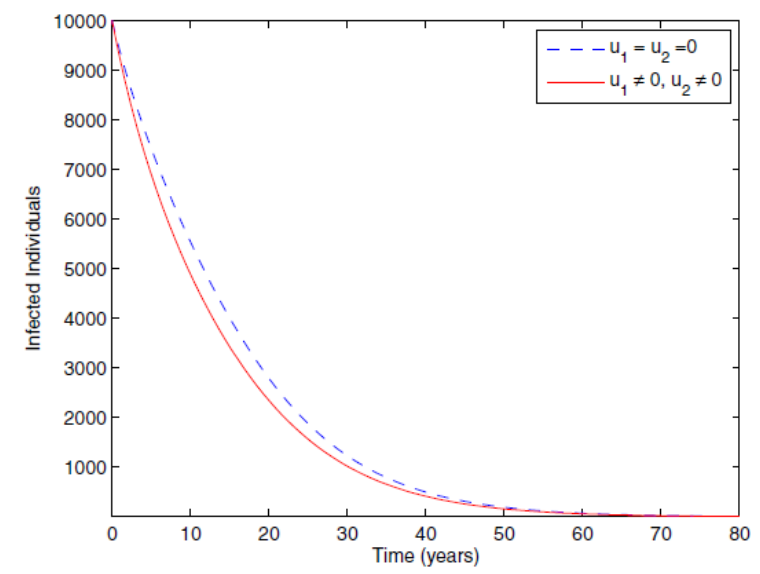

(a)

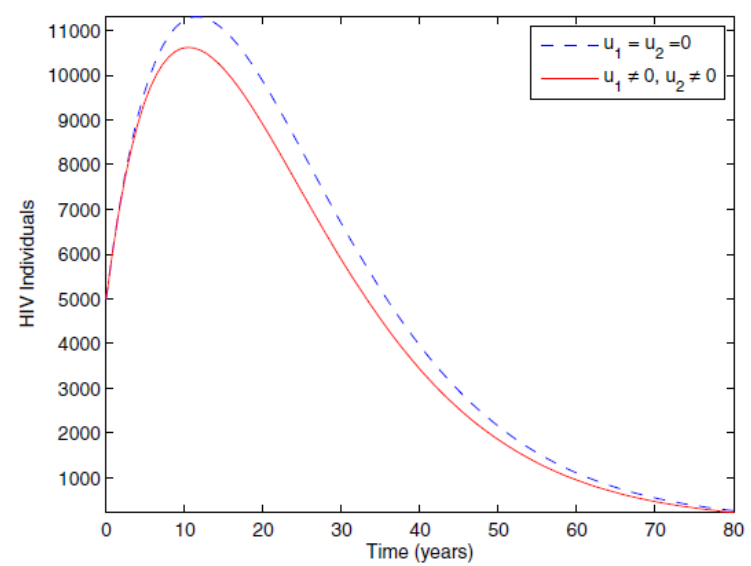

(b)

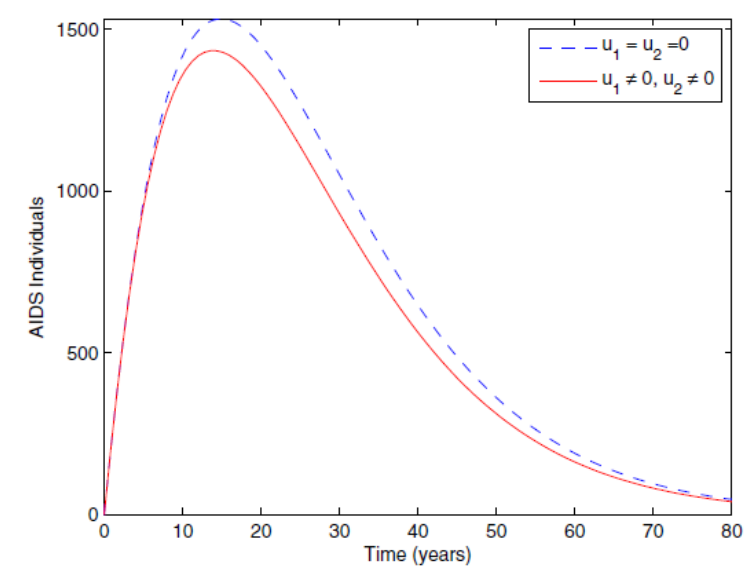

(c)

Fig. 3. Simulations of HIV/AIDS model showing the effect of optimal control strategies using only screening control (without parental care) on individuals. but over 80 when there is no control. The control profile indicates that maximum effort is required on screening control $\left(u_{1}\right)$ until almost the very end of the period of interest, while parental care $\left(u_{2}\right)$ can be relaxed slightly earlier. The graph of the controls are otherwise uninformative in appearance and is therefore not shown.

\section{Strategy B: The use of only screening control $\left(u_{1}\right)$ on individuals.}

Here, only screening control $u_{1}$ is used to optimize the objective function $J$ while we set the parental care $\left(u_{2}\right)$ to zero. We observe a minor difference between the controlled and uncontrolled cases. It is also noted that there is a rapid increase in population of HIV individuals and AIDS individuals in Figs. 3(b) and 3(c) respectively.

Strategy C: The use of only parental care $\left(u_{2}\right)$ on individuals. Simulations here are similar to that of strategy $A$ with only slight differences. Only the parental care $(u)$ is used to optimize the objective function $J$ while screening control $\left(u_{1}\right)$ is set to zero. We observe in Fig. 4(a) that due to control strategies, the 


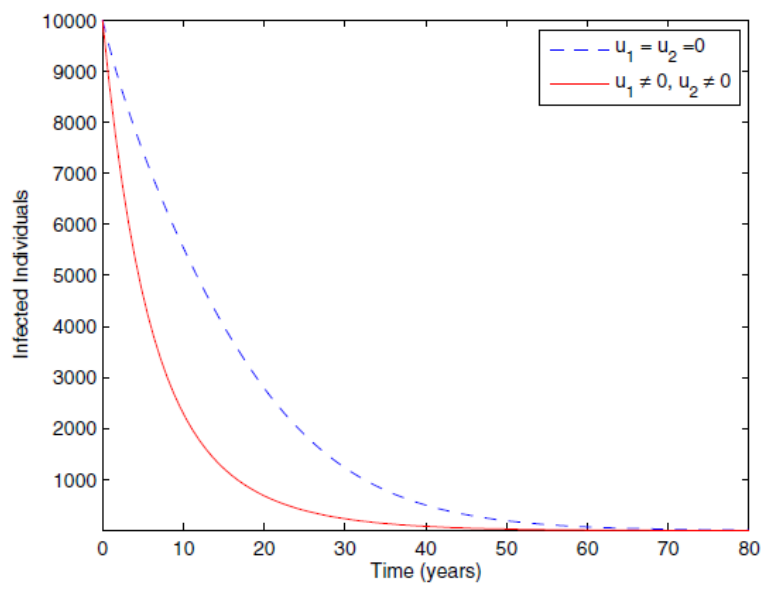

(a)

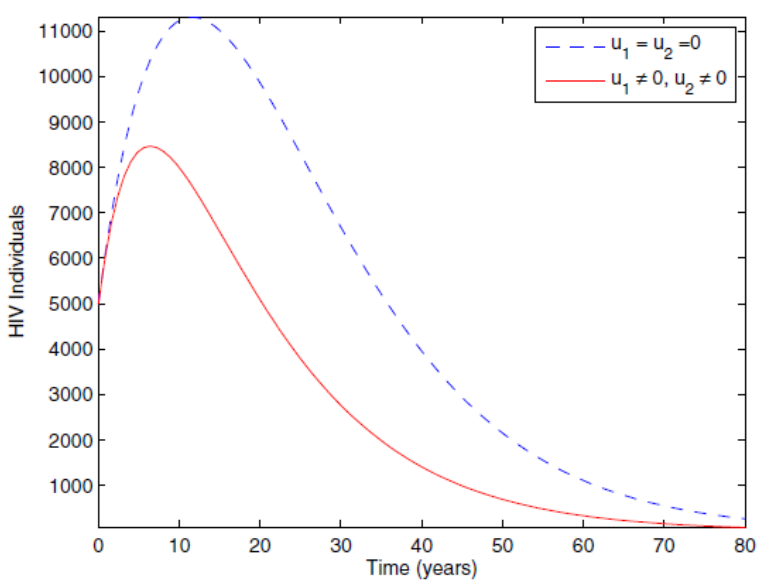

(b)

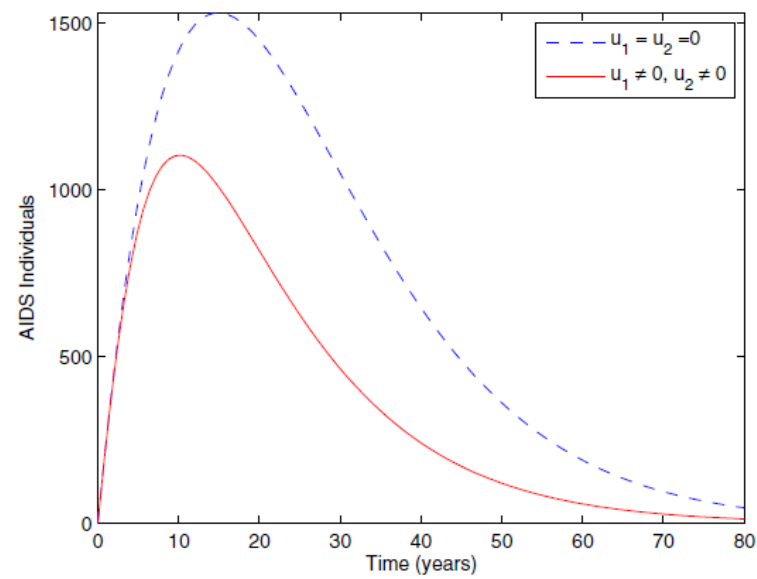

(c)

Fig. 4. Simulations of HIV/AIDS model showing the effect of optimal control strategies using only parental care (without screening control) on individuals.

number of infected individuals $I$ decreases to zero at approximately time $t=48$. In the uncontrolled case $I$ approaches zero at $t=68$. Similarly in Fig. 4(b), the number of HIV individuals also increases to 8500 from an initial 5000 but later decreases to zero at $t=$ 73 when there is parental care. However, $H$ rapidly increases to 11,000 when there is no control, and reduces to reach 300 when $t=80$. AIDS individuals in Fig. 4(c) increases from initial $\mathrm{o}$ to approximately 1200 and decreases to 1 at $t=80$ when there is parental care, but goes over 1500 and later reduces to 50 at $t=80$ when there is no parental care.

Simulations from strategy $A, B$ and $C$ show that parental care $\left(u_{2}\right)$ is more effective than screening control $\left(u_{1}\right)$ in controlling HIV/AIDS, but a combination of both controls as in strategy $A$ gives the best result.

\section{Conclusion}

In this study, we proposed a deterministic population model of HIV/AIDS for studying the effect of parental care when we have infected immigrant youths. We analysed the model for 
the existence of disease-free and endemic equilibrium points, and we found the model to exhibit good stability properties. In particular, the model does not have a disease-free equilibrium in the presence of immigration of infected youths. However, in the case of no recruitment of infectives, the disease-free equilibrium is globally asymptotically stable while $R_{\mathrm{O}}<1$. The sensitivity analysis, with the given parameter values, shows that the two most sensitive parameters are the number of contacts with AIDS individuals $\left(c_{3}\right)$, and the transmission probability of getting AIDS $\left(\beta_{3}\right)$. This type of information is useful when planning for intervention.

We derived and analyzed the conditions for optimal control of the disease with screening control and parental care on youths. The control profile revealed that maximum effort is required on screening control through the intervention, while parental care can be relaxed after a period of time. We further found that if there is effective parental care or control over the youths, then infected immigrants do not have a strong impact on the disease transmission. We identified the combination of screening of immigrants and parental care that gives the most efficient results in controlling the spread of HIV/AIDS. The adjoint variables reveal that the cost implications on susceptible and AIDS individuals are much less than on infected and HIV individuals. Therefore control programs that follow these strategies will optimally reduce the spread of HIV/AIDS.

Research [4] of Ayankogbe et al., for instance, shows that AIDS prevention campaigns should be based on a diverse basket of intervention types rather than being focused on just one type of intervention. There is a need for models to consider factors such as culture and religion and the extent to which they may be utilized to reduce the spread of HIV/AIDS. Proper consideration of these and other factors will provide a better understanding of the population dynamics of the disease and its control.

\section{Acknowledgment}

P. J. Witbooi acknowledges funding from the National Research Foundation of South Africa. 


\section{References}

[1] B.M. Adams,H. T. Banks,H.Kwon and H. T. Tran, Dynamic multidrugtherapies for HIV: Optimal and STI control approaches, Math. Biosci. Engrg. 1(2) (2004) 223-241. [2] R. M. Anderson, The role of mathematical models in the study of HIV transmission and the epidemiology of AIDS, J. Acquired Immun. Def. Syndrome 1 (1988) 241-256.

[3] R. M. Anderson, G. F. Medly, R. M. May and A. M. Johnson, A preliminary study of the transmission dynamics the Human Immunodeficiency Virus (HIV), the causative agent of AIDS, IMA J. Math. Appl. Med. Biol. 3 (1986) 229-263.

[4] O. O. Ayankogbe, K. Odusote, M. O. Omoegun, V. Ofoha, A. Adedokun and K. O. Abiola, Determinants of young peoples' sexual behaviour concerning HIV and AIDS in the practice population of a university health centre in Lagos, Nigeria, Afr. $J$. Prim. Health Care Fam. Med. 3(1) (2011), Art. 219, 8pp., doi: 10.4102/http://www.phcfm.org phcfm.v3i1.219.

[5] C. P. Bhunu, S. Mushayabasa, H. Kojouharov and J. M. Tchuenche, Mathematical analysis of an HIV/AIDS model: Impact of educational programs and abstinence in subSaharan Africa, J. Math. Model. Algorithms 10(1) (2011) 31-55.

[6] L. Cai, L. Li, M. Ghosh and B. Guo, Stability analysis of an HIV/AIDS epidemic model with treatment, J. Comput. Appl. Math. 229 (2009) 313-323.

[7] N. Dalal, D. Greenhalgh and X. Mao, A stochastic model of AIDS and condom use, J. Math. Anal. Appl. 325 (2007) 36-53.

[8] Effects of Poor Parental Care on Child Development, http://ezinearticles.com/ Effects-of-Poor-Parental-Care-on-Child-Development, and http://EzineArticles.com/ 1947034.

[9] H. R. Joshi, Optimal control of an HIV immunology model, Optim. Control Appl.

Meth. 23 (2002) 199-213.

[10] S. Lenhart and J. Workman, Optimal Control Applied to Biological Models,Mathe- matical and Computational Biology Series (Chapman and Hall/CRC, London, UK, 2007).

[11] R. M. May and R. M. Anderson, Transmission dynamics of HIV infection, Nature 3426 (1987) 137-142.

[12] Z. Mukandavire, K. Bowa and W. Garira, Modelling circumcision and condom use as HIV/AIDS preventive control strategies, Math. Comput. Model. 46 (2007) 13531372.

[13] Z. Mukandavire, C. Chiyaka, G. Magombedze, G. Musuka and N. J. Malunguza, Assessing the effects of homosexuals and bisexuals on the intrinsic dynamics of HIV/AIDS in heterosexual settings, Math. Comput. Model. 49 (2009) 1869-1882.

[14] Z. Mukandavire and W. Garira, Effects of public health educational campaigns and the role of sex workers on the spread of HIV/AIDS among heterosexuals, Theor. Popul. Biol. 72 (2007) 346-365.

[15] Z. Mukandavire, W. Garira and C. Chiyaka, Asymptotic properties of an HIV/AIDS model with delay, J. Math. Anal. Appl. 330 (2009) 961-933. 
[16] Z. Mukandavire, W. Garira and J. Tchuenche, Modeling effects of public health educational campaigns on HIV/AIDS transmission dynamics, Appl. Math. Model. 33 (2009) 2084-2095.

[17] R. Naresh, A. Tripathi and D. Sharma, Modelling the effect of screening of unaware infectives on the spread of HIV infection, Appl. Math. Comput. 184 (2007) 10531068.

[18] F. Nyabadza, C. Chiyaka, Z. Mukandavire and S. D. Hove-Musekwa, Analysis of an HIV/AIDS model with public-health information campaigns and individual with- drawal, J. Biol. Syst. 18(2) (2010) 357-375.

[19] UN, UNAIDS, World Health Organization, 2011 AIDS epidemic update available, http://news.yahoo.com/s/afp/20110603/hl afp/healthaidsanniversary-unaids 20110603181329 (November 2011).

[20] P. van den Driessche and J. Watmough, Reproduction numbers and subthreshold endemic equilibria for compartmental models of disease transmission, Math. Biosci. 180 (2002) 29-48. 